\title{
The Method of Fundamental Solutions with Eigenfunction Expansion Method for Nonhomogeneous Diffusion Equation
}

\author{
D. L. Young, ${ }^{1}$ C. W. Chen, ${ }^{1}$ C. M. Fan, ${ }^{1}$ C. C. Tsai ${ }^{2}$ \\ ${ }^{1}$ Department of Civil Engineering and Hydrotech Research Institute, National Taiwan \\ University, Taipei, Taiwan \\ ${ }^{2}$ Department of Information Technology, Toko University, Chia-Yi County, Taiwan
}

Received September 2005; accepted November 2005

Published online 27 January 2006 in Wiley InterScience (www.interscience.wiley.com).

DOI 10.1002/num.20148

\begin{abstract}
In this article we describe a numerical method to solve a nonhomogeneous diffusion equation with arbitrary geometry by combining the method of fundamental solutions (MFS), the method of particular solutions (MPS), and the eigenfunction expansion method (EEM). This forms a meshless numerical scheme of the MFS-MPS-EEM model to solve nonhomogeneous diffusion equations with time-independent source terms and boundary conditions for any time and any shape. Nonhomogeneous diffusion equation with complex domain can be separated into a Poisson equation and a homogeneous diffusion equation using this model. The Poisson equation is solved by the MFS-MPS model, in which the compactly supported radial basis functions are adopted for the MPS. On the other hand, utilizing the EEM the diffusion equation is first translated to a Helmholtz equation, which is then solved by the MFS together with the technique of the singular value decomposition (SVD). Since the present meshless method does not need mesh generation, nodal connectivity, or numerical integration, the computational effort and memory storage required are minimal as compared with other numerical schemes. Test results for two 2D diffusion problems show good comparability with the analytical solutions. The proposed algorithm is then extended to solve a problem with irregular domain and the results compare very well with solutions of a finite element scheme. Therefore, the present scheme has been proved to be very promising as a meshfree numerical method to solve nonhomogeneous diffusion equations with time-independent source terms of any time frame, and for any arbitrary geometry. (C) 2006 Wiley Periodicals, Inc. Numer Methods Partial Differential Eq 22: 1173-1196, 2006
\end{abstract}

Keywords: method of fundamental solutions; method of particular solutions; eigenfunction expansion method; singular value decomposition; diffusion equation

\section{INTRODUCTION}

There are many physical processes governed by the diffusion equation, such as fluid flows, solute transports, heat transfers, chemical and biologic processes, and others. Although for some

Correspondence to: D. L. Young, Department of Civil Engineering and Hydrotech Research Institute, National Taiwan University, Taipei, Taiwan (email: dlyoung @ntu.edu.tw)

Contract grant sponsor: National Science Council of Taiwan; contract grant number: NSC 93-2611-E-002-001.

() 2006 Wiley Periodicals, Inc. 
simplified situations the analytical solutions may be available, the complicated geometry generally requires employing numerical methods. Classical numerical schemes such as the finite difference method (FDM), finite element method (FEM), finite volume method (FVM) and boundary element method (BEM) have been extensively utilized to solve different kinds of diffusion problems. For example, Hobson et al. [1] and Chawla and Al-Zanaidi [2] both applied the FDM to solve the diffusion equation. Moreover, Oden et al. [3] employed the FEM to solve the diffusion process in an unbounded medium. On the other hand, Jones and Menzies [4] used the FVM for the diffusion equation. Also, Zhu [5], Zhu et al. [6], Bulgakov et al. [7], Zerroukat [8], Sutradhar et al. [9], and Bialecki et al. [10] applied the BEM to solve diffusion equation problems. However, by using these classical domain dominated methods, the mesh generation may require a significant computational effort and a large amount of memory storage to implement complex geometry, especially in $3 \mathrm{D}$ problems.

Considerable computational effort is necessary to compute the domain integration for the source term when applying the traditional BEM. Nardini and Brebbia [11] were the first to introduce the dual reciprocity method (DRM) in BEM to transform the domain integral to a boundary type by a series of radial basis functions (RBFs). On the other hand, Golberg and Chen [12] extended the method of fundamental solutions (MFS) with the method of particular solutions (MPS) to deal with the particular solutions for the partial differential equations. In the meantime they also showed that the DRM and the MPS are equivalent in the Poisson equation. The combined MFS-MPS model is free from the singular integral evaluation for solving nonhomogeneous linear operators, as is generally required by the BEM. Therefore, the MFS-MPS model, as a meshless numerical algorithm, has been considered to be a successful solver for many diffusion problems explained in the following.

From the literature review, we found that the solutions of diffusion equations used either the finite difference scheme $[12,13]$ or Laplace transform $[14,15]$ to deal with the time derivative term before utilizing the MFS. This is due to the fact that the MFS is well treated in the spatial domain after the treatment of the transient part. In the work of Chen et al. [15], they used the Laplace transform to change the diffusion equation to a modified Helmholtz equation with source term and then used the MFS-DRM to solve the modified Helmholtz equation. The inverse transform, which sometimes leads to certain difficulties in the solution process, would be needed after the adoption of the Laplace transform. When the time derivative is discretized by the finite difference scheme, the same drawback incurred in the Laplace transform is encountered, since it also eventually results in the modified Helmholtz equation with source term. Moreover, the particular solution of the modified Helmholtz operator is mathematically more difficult than the particular solution of the nonhomogeneous diffusion operator, in which only the inverse Laplace operator is required, if the source terms and boundary conditions are taken time-independent.

To overcome the drawbacks of the Laplace transform and finite difference time discretization, the time-dependent MFS with space-time collocation method was recently introduced by Young et al. [16] to solve multidimensional diffusion equations. Young et al. [17] further extended the time-dependent diffusion MFS-DRM model to solve multidimensional nonhomogeneous diffusion problems. However there is another way, completely different from the abovementioned methods, to treat the time derivative, that is, the MFS-EEM model. Yao and Margrave [18] have already used the eigenfunction transform method to solve the wave equation. As far as time evolution is concerned, once the geometry is chosen, the EEM is more feasible and robust for the transient calculation of any time. Furthermore, the diffusion and wave equations are treated almost identically by using the MFS-EEM model. Therefore, the present model will provide a very versatile vehicle to generalize the concept of the orthogonal eigenfunction expansion to multiple dimensions in any shape of geometry.

Numerical Methods for Partial Differential Equations DOI 10.1002/num 
In this article, the MFS-MPS-EEM model with the singular value decomposition (SVD) method is adopted to solve a nonhomogeneous diffusion equation. The nonhomogeneous diffusion equation is separated into the Poisson equation and the homogeneous diffusion equation without the need of Laplace transform or finite difference or space-time collocation methods as in the articles cited above. The time-dependent solutions are directly solved by the superposition principle of the eigenvalues and eigenfunctions obtained by the MFS with SVD. Depending on the magnitude of the diffusivity, only very few eigenfunctions are physically needed to represent the solutions for diffusion problems. Moreover, initial condition is used to determine the weighting coefficients of the orthogonal eigenfunctions. As a first attempt, the model is applied to test a set of 2D diffusion problems with Dirichlet boundary conditions. To compare the accuracy of the present method, analytical solutions are used. The article is organized as follows. In Section 2 the governing equation and the initial and boundary conditions considered are described. The numerical discretization of the MFS, MPS, EEM, and SVD schemes are elaborated in Section 3. In Section 4 we delineate the comparisons of the present results with two analytical solutions and one with the FEM computation for the three test cases. The final conclusions of this study are given in Section 5 .

\section{GOVERNING EQUATION}

Consider a nonhomogeneous diffusion equation with a time-independent source term and boundary conditions (BC) over a computational domain $\Omega$ with boundary $\Gamma$,

$$
\frac{\partial u(\mathbf{x}, t)}{\partial t}=k \nabla^{2} u(\mathbf{x}, t)+A(\mathbf{x}),
$$

in which $\mathbf{x}$ is the general spatial coordinate, $t$ is the time, $k$ is the diffusion coefficient (diffusivity), $A(\mathbf{x})$ is the time-independent source function, and $u(\mathbf{x}, t)$ is the scalar variable to be determined. The initial condition (IC) of the diffusion equation is

$$
u(\mathbf{x}, 0)=B(\mathbf{x}) \quad \text { in } \Omega
$$

with the Dirichlet and/or Neumann BC:

$$
\begin{aligned}
u(\mathbf{x}, t) & =C(\mathbf{x}) & & \text { on } \Gamma^{1} \\
\frac{\partial u}{\partial n}(\mathbf{x}, t) & =D(\mathbf{x}) & & \text { on } \Gamma^{2},
\end{aligned}
$$

where $\Gamma^{1}+\Gamma^{2}$ is equal to the boundary $\Gamma$ and $n$ is the outward normal direction. Moreover, the $\mathrm{BC}$ is of Dirichlet type if only $\Gamma^{2}=0$, of Neumann type if only $\Gamma^{1}=0$, and of Robin type if both $\Gamma^{1} \neq 0$ and $\Gamma^{2} \neq 0$. For simplicity, the boundary conditions $C(\mathbf{x})$ and $D(\mathbf{x})$ are assumed to be time-independent functions. The augmented data of the problem are $A(\mathbf{x}), B(\mathbf{x}), C(\mathbf{x})$, and $D(\mathbf{x})$, which are all time-independent known functions.

\section{NUMERICAL METHOD}

Based on the superposition principle of the linear system, nonhomogeneous diffusion problems with time-independent source terms and BCs can be separated into a Poisson equation and a 
homogeneous diffusion equation. According to the superposition principle, the solution of the nonhomogeneous diffusion equation will be represented as follows:

$$
u(\mathbf{x}, t)=u_{1}(\mathbf{x})+u_{2}(\mathbf{x}, t) \quad \text { in } \Omega,
$$

where $u_{1}(\mathbf{x})$ satisfies the Poisson equation,

$$
\begin{array}{rlrl}
\nabla^{2} u_{1}(\mathbf{x}) & =-\frac{1}{k} A(\mathbf{x}) & \\
u_{1}(\mathbf{x}) & =C(\mathbf{x}) & & \text { on } \Gamma^{1} \\
\frac{\partial}{\partial n} u_{1}(\mathbf{x}) & =D(\mathbf{x}) & & \text { on } \Gamma^{2}
\end{array}
$$

and $u_{2}(\mathbf{x}, t)$ satisfies the homogeneous diffusion equation,

$$
\begin{aligned}
\frac{\partial u_{2}(\mathbf{x}, t)}{\partial t} & =k \nabla^{2} u_{2}(\mathbf{x}, t) \\
u_{2}(\mathbf{x}, 0) & =B(\mathbf{x})-u_{1}(\mathbf{x}) \quad \text { in } \Omega \\
u_{2}(\mathbf{x}, t) & =0 \quad \text { on } \Gamma^{1} \\
\frac{\partial}{\partial n} u_{2}(\mathbf{x}, t) & =0 \quad \text { on } \Gamma^{2}
\end{aligned}
$$

In Eq. (5), $u_{1}(\mathbf{x})$ is a time-independent function that physically represents a steady state (or quasistatic) solution $[17,19]$. Then, from the superposition principle we can solve Poisson equation (5) by decomposing the solution into homogenous and particular solutions as follows:

$$
u_{1}(\mathbf{x})=u_{h}(\mathbf{x})+u_{p}(\mathbf{x}),
$$

where $u_{h}(\mathbf{x})$ is the homogenous solution and $u_{p}(\mathbf{x})$ is the particular solution. In which the particular solution, $u_{p}(\mathbf{x})$ satisfies

$$
\nabla^{2} u_{p}(\mathbf{x})=-\frac{1}{k} A(\mathbf{x})
$$

On the other hand, the homogenous solution, $u_{h}(\mathbf{x})$, satisfies the Laplace equation as well as the modified BC:

$$
\begin{aligned}
\nabla^{2} u_{h}(\mathbf{x}) & =0 \quad \text { in } \Omega \\
u_{h}(\mathbf{x}) & =C(\mathbf{x})-u_{p}(\mathbf{x}) \quad \text { on } \Gamma^{1} \\
\frac{\partial}{\partial n} u_{h}(\mathbf{x}) & =D(\mathbf{x})-\frac{\partial}{\partial n} u_{p}(\mathbf{x}) \quad \text { on } \Gamma^{2}
\end{aligned}
$$

The particular solution corresponding to Eq. (8) can be approximated by the MPS for the source term $-(1 / k) A(\mathbf{x})$

$$
\begin{aligned}
-\frac{1}{k} A(\mathbf{x}) & =\sum_{j=1}^{N} a_{j}^{p} f_{j}(r) \\
f(r) & = \begin{cases}\left(1-\frac{r}{\alpha}\right)^{2}, & r \leq \alpha \\
0, & r>\alpha,\end{cases}
\end{aligned}
$$


where $f(r)$ is the CSRBFs [20], $\alpha$ is the compact radius, $r=\left|\mathbf{x}-\mathbf{x}_{j}\right|$ is the radial distance between the $j$ th field point $\mathbf{x}_{j}$ and the collocation point $\mathbf{x}$, and $N$ is the number of collocation nodes. In this study the collocation points are typically distributed in the interior domain as well as on the boundary (Fig. 1). After applying Eq. (10) at $N$ collocation points, the unknown coefficient $a_{j}^{p}$ 's can be solved [21]. Therefore, the particular solution $u_{p}(x)$ is determined by inverting the Laplace operator of Eq. (8) [22-24]:

$$
\begin{aligned}
& u_{p}(\mathbf{x})=\sum_{j=1}^{N} \alpha_{j}^{p} F_{j}(r)
\end{aligned}
$$

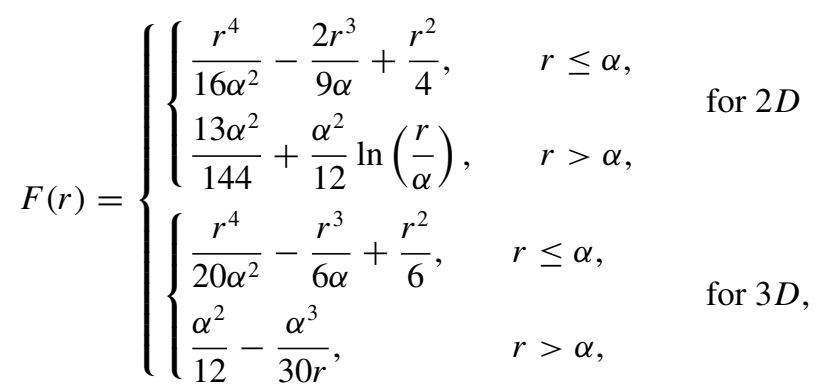

where $F(r)$ is the corresponding CSRBFs of $f(r)$ in Eq. (10). With the substitution of Eq. (11) into the modified boundary conditions of the homogenous Eq. (9), the results will be a well-posed

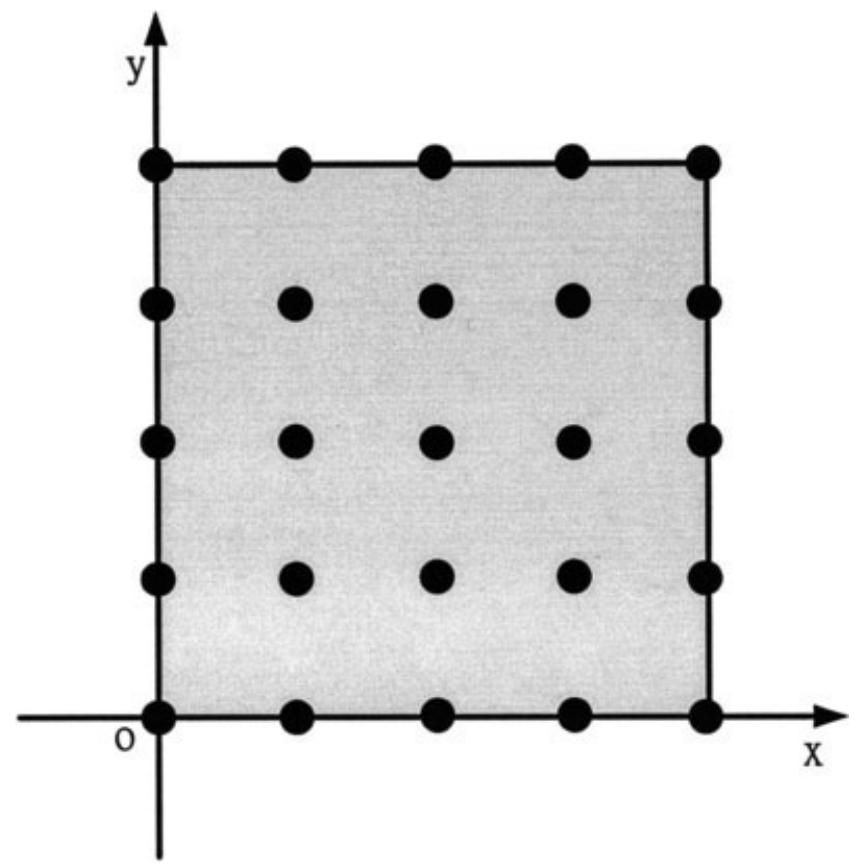

FIG. 1. Collocation points for the MPS. 
Laplace equation. The MFS is then applied to solve the Laplace equation:

$$
-\nabla^{2} G_{1}(r)=\delta(\mathbf{x})
$$

where $G_{1}(r)$ is the fundamental solution of the Laplace equation, which can be obtained by the potential theory. Then the solution of Laplace equation is represented by the MFS form as described by the following:

$$
\begin{aligned}
& u_{h}(\mathbf{x})=\sum_{j=1}^{M} \alpha_{j}^{h} G_{1 j}(r) \\
& G_{1}(r)= \begin{cases}-\frac{1}{2 \pi} \ln (r), & \text { for } 2 D, \\
\frac{1}{4 \pi r}, & \text { for } 3 D,\end{cases}
\end{aligned}
$$

where $r=\left|\mathbf{x}-\mathbf{x}_{j}\right|$ is the distance between the field point $\mathbf{x}$ and the $j$ th source point $\mathbf{x}_{j}, M$ is the number of source nodes. So the solution $u_{1}(\mathbf{x})$ of the Poisson equation is then obtained by MFS-MPS model.

After having applied the MFS-MPS model for the Poisson equation, we ultimately utilize the MFS-EEM to solve the homogenous diffusion equation. For simplicity, we now omit the summation of the infinite series of the eigenfunction expansion at this moment. Let $u_{2}(\mathbf{x}, t)=$ $u_{s}(\mathbf{x}) e^{-\lambda^{2} k t}$ and substitute into Eq. (6), we obtain:

$$
\nabla^{2} u_{s}(\mathbf{x})+\lambda^{2} u_{s}(\mathbf{x})=0 .
$$

Therefore, the homogeneous diffusion equation in Eq. (6) is transformed to the Helmholtz equation in Eq. (14) with homogeneous boundary conditions. Since we have to face the homogeneous boundary conditions, the above eigenvalue problem is solved by the MFS with the SVD method. The fundamental solution of the Helmholtz equation satisfies the following equation:

$$
-\left(\nabla^{2}+\lambda^{2}\right) G_{2}(r)=\delta(\mathbf{x})
$$

where $G_{2}(r)$ is the fundamental solution of the Helmholtz equation, which can be obtained by mathematical analysis. Then the solution of the Helmholtz equation is obtained by the MFS as described below:

$$
\begin{array}{rlrl}
u_{s}(\mathbf{x}) & =\sum_{j=1}^{Q} \beta_{j} G_{2 j}(r) & \\
G_{2}(r) & = \begin{cases}\frac{-i}{4} H_{0}^{(2)}(\lambda r), & \text { for } 2 D, \\
\frac{1}{4 \pi r} e^{-i \lambda|r|}, & \text { for } 3 D,\end{cases} \\
u_{s}(\mathbf{x}) & =0 \quad \text { on } \Gamma^{1} \\
\frac{\partial}{\partial n} u_{s}(\mathbf{x}) & =0 \quad \text { on } \Gamma^{2},
\end{array}
$$


where $H_{0}^{(2)}$ ( ) is the Hankel function of the second kind of order zero, $r=\left|\mathbf{x}-\mathbf{x}_{j}\right|$ is the distance between the field point $\mathbf{x}$ and the $j$ th source point $\mathbf{x}_{j}, Q$ is the number of source nodes. Since Eq. (16) has nontrivial solutions only for some discrete eigenvalues, we use the MFS with SVD [25] to obtain imbedding eigenvalues, $\lambda$, and corresponding eigenfunctions, $u_{s}(\mathbf{x})$, for Eq. (16). Then the major advantage of the orthogonal eigenfunction expansion method is beneficial to this study. The modified initial condition of the homogenous diffusion equation is obtained by the following eigenfunction expansion formula:

$$
u_{2}(\mathbf{x}, 0)=B(\mathbf{x})-u_{1}(\mathbf{x})=\sum_{j=1}^{\infty} \gamma_{j} u_{s_{j}}(\mathbf{x}),
$$

where $u_{s_{j}}(\mathbf{x})$ is the $j$ th eigenfunction with corresponding eigenvalue $\lambda_{j}, \gamma_{j}$ is the weighting coefficient of every eigenfunction which is determined by collocating the modified initial conditions (17) at some finite interpolating points inside the domain. Depending on the diffusivity magnitude, the first few eigenfunctions are physically enough to represent the solutions for the diffusion problems. In our numerical test, the finite terms are used to replace the infinite series in Eq. (17) and the results show that the assumption is acceptable:

$$
u_{2}(\mathbf{x}, 0)=B(\mathbf{x})-u_{1}(\mathbf{x})=\sum_{j=1}^{P} \gamma_{j} u_{s_{j}}(\mathbf{x}),
$$

where $P$ is the number of adopted eigenfunctions. Therefore, the EEM is capable of obtaining time-dependent solutions without using the time-marching or space-time collocation methods.

From Eqs. (4)-(18), the solutions of Eq. (1) are achieved by the linear superposition:

$$
\begin{aligned}
u(\mathbf{x}, t) & =u_{1}(\mathbf{x})+u_{2}(\mathbf{x}, t) \\
& =u_{h}(\mathbf{x})+u_{p}(\mathbf{x})+u_{2}(\mathbf{x}, t) \\
& =\sum_{j=1}^{M} \alpha_{j}^{h} G_{1 j}(r)+\sum_{j=1}^{N} \alpha_{j}^{p} F_{j}(r)+\sum_{j=1}^{P} \gamma_{j} u_{s_{j}}(r) e^{-\lambda_{j}^{2} k t}
\end{aligned}
$$

Thus the solutions of the nonhomogeneous diffusion equations with time-independent source terms and boundary conditions can be solved by the proposed scheme.

\section{RESULTS AND DISCUSSION}

Validation for the proposed numerical method, the MFS-MPS-EEM model, is achieved by comparing the results with the analytical solutions for diffusion problems with Dirichlet BC. The effectiveness of the proposed method is verified by solving $2 \mathrm{D}$ homogeneous and nonhomogeneous diffusion problems. In the following sections we will discuss the results. Figure 1 shows the collocation points of the 2D MPS for the nonhomogeneous solutions. On the other hand, the source points and the field points of the MFS based on the fundamental solution of the linear equation are depicted in Fig. 2 for a 2D problem. In the numerical experiments (Fig. 3), both regular domains (Example 1: circle; Example 2: rectangle) and an irregular domain (Example 3) are considered. 


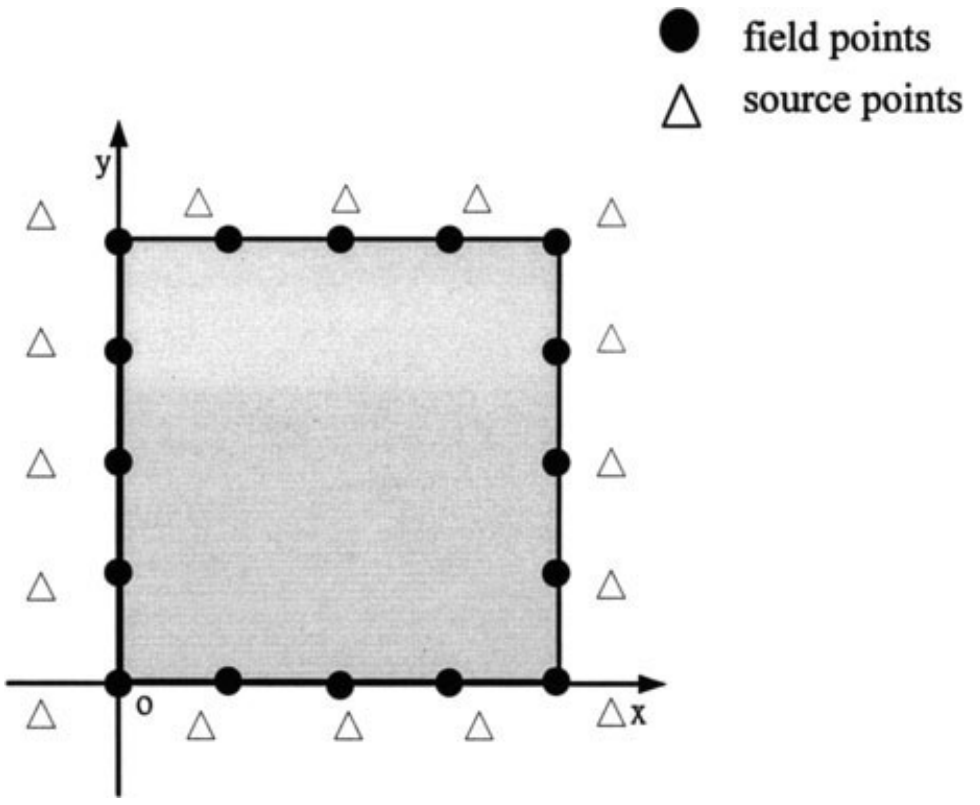

FIG. 2. Schematic diagram of source and field points for the MFS.

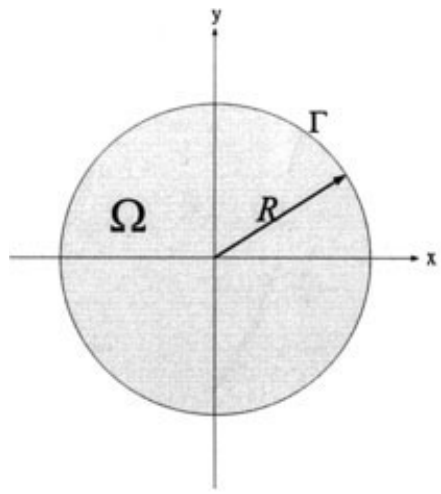

(a)

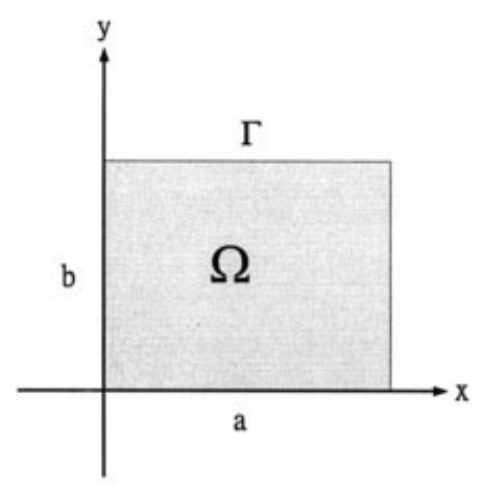

(b)

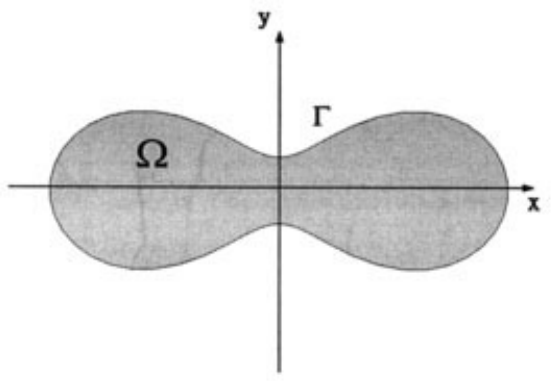

(c)

FIG. 3. Schematic diagram of (a) circular cavity, (b) rectangular cavity, and (c) an Oval of Cassini. 
Example 1. Consider a unit circular disk [Fig. 3(a)] that is independent of $\theta$, satisfies the following governing diffusion equation (DE) with IC and BC.

$$
\begin{array}{lll}
\text { DE } & k \frac{1}{r} \frac{\partial}{\partial r}\left(r \frac{\partial u}{\partial r}\right)=\frac{\partial u}{\partial t} \\
\text { IC } & u(r, 0)=1 & \text { in } \Omega \\
\text { BC } & u(1, t)=0 & \text { on } \Gamma^{1}
\end{array}
$$

The analytical solution of the problem is given by

$$
u(r, t)=\sum_{n=1}^{\infty} \frac{2 J_{0}\left(\lambda_{n} r\right)}{\lambda_{n} J_{1}\left(\lambda_{n}\right)} e^{-\lambda_{n}^{2} k t},
$$

where $J_{0}\left(\lambda_{n}\right)=0$ is the solution of the eigenvalues, $J_{0}\left(\right.$ ) and $J_{1}($ ) are the Bessel functions of the first kind of the order zero and one, respectively. The first five calculated eigenvalues $\lambda_{n}$ and eigenfunctions by MFS-EEM with SVD model are shown in Fig. 4. These numerically obtained eigenvalues are almost the same as the exact solutions of $J_{0}\left(\lambda_{n}\right)=0$. Comparison shows that the differences of eigenvalues between the computed and analytical results are only after five digits. The comparisons between the solutions of the MFS-EEM with SVD model and the analytical solutions of the u-distribution along $x=0$ for different time levels are depicted in Fig. 5. Though only five eigenfunctions and 961 points to interpolate eigenfunctions are used in the numerical modeling, there is not much deviation from the analytical solutions. The full-field distributions with time evolution are shown in Fig. 6. The results generally exhibit good agreements with the analytical solutions at different time stages. The variations of the time decay clearly demonstrate the physics underlying the diffusion process. Moreover, Figs. 7 and 8 show the time evolution history at $(0.5,0)$ of the solution and the maximum relative error of the computational domain. On the other hand, Fig. 9 depicts the maximum relative error histograms when using the present method for different numbers of points to interpolate eigenfunctions, when, as expected, more points generally will render better resolution. If the diffusivity is not too small, physically the first few eigenfunctions are almost qualified to represent the diffusion solution. In Fig. 10, the comparison of the time history of the maximum relative error for different numbers of eigenfunctions is described when 961 points are used to interpolate eigenfunctions. The advantage of the method is the capability to obtain the solution for any time by the superposition principle of the first few eigenfunctions, in which their weighting coefficients are dependent on IC.

Example 2. After simulating the circular domain, the proposed method is utilized to study the second example in a rectangular slab of size $[0, a] \times[0, b]$ [Fig. 3(b)], a 2D diffusion problem with nonhomogeneous boundary conditions and nonhomogeneous sources:

$$
\begin{array}{ll}
\text { DE } & \left(\frac{\partial^{2} u}{\partial x^{2}}+\frac{\partial^{2} u}{\partial y^{2}}\right)-6 x+2 \sin x \sin y=\frac{1}{k} \frac{\partial u}{\partial t} \\
\text { IC } & u(x, y, 0)=x y+x^{3}+\sin x \sin y \quad \text { in } \Omega \\
\text { BC } & u(x, y, t)=x^{3}+\sin x \sin y \quad \text { on } \partial \Gamma^{1} .
\end{array}
$$


YOUNG ET AL.

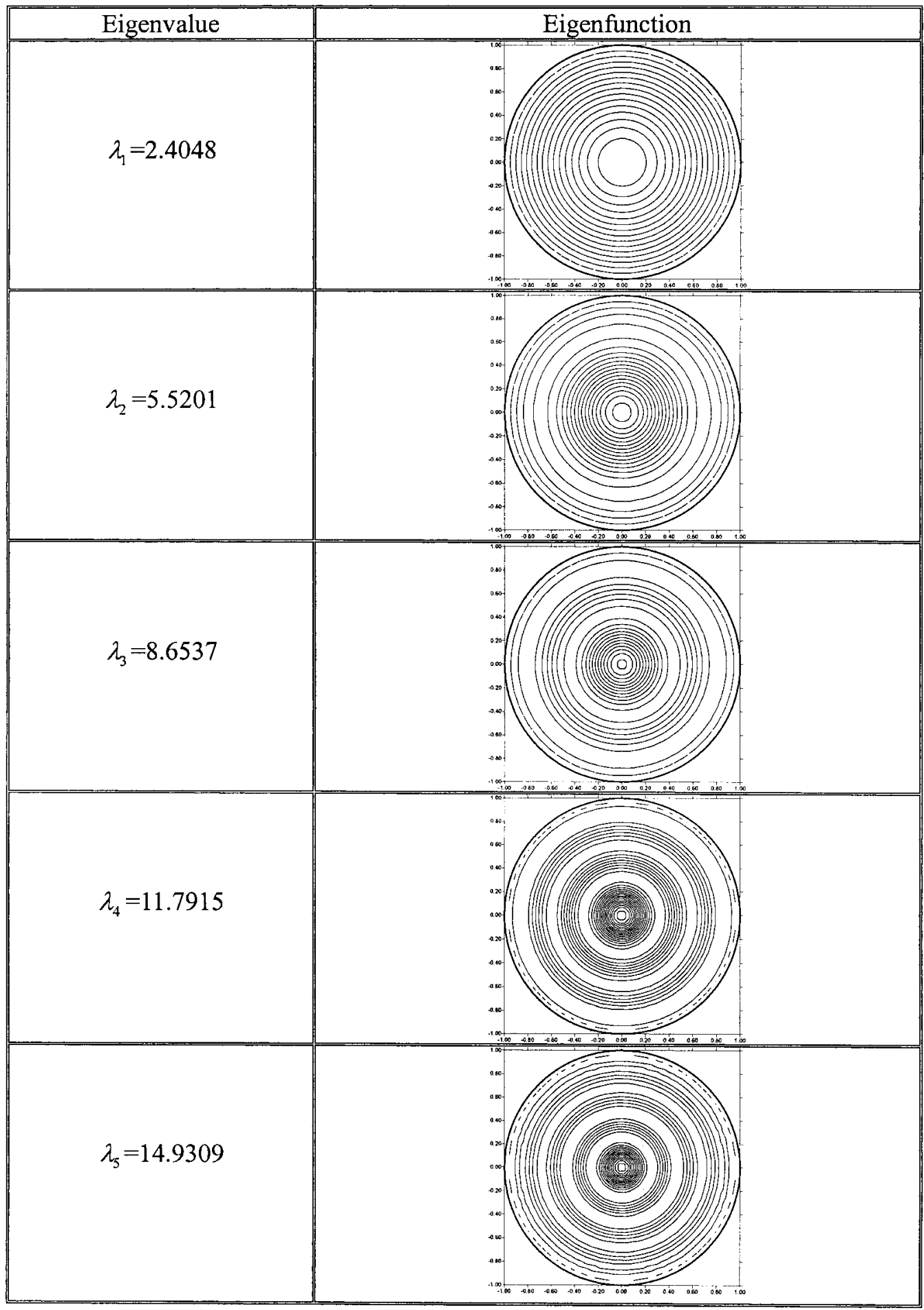

FIG. 4. The first 5 eigenvalues and eigenfunctions for circular problem for Example 1. 


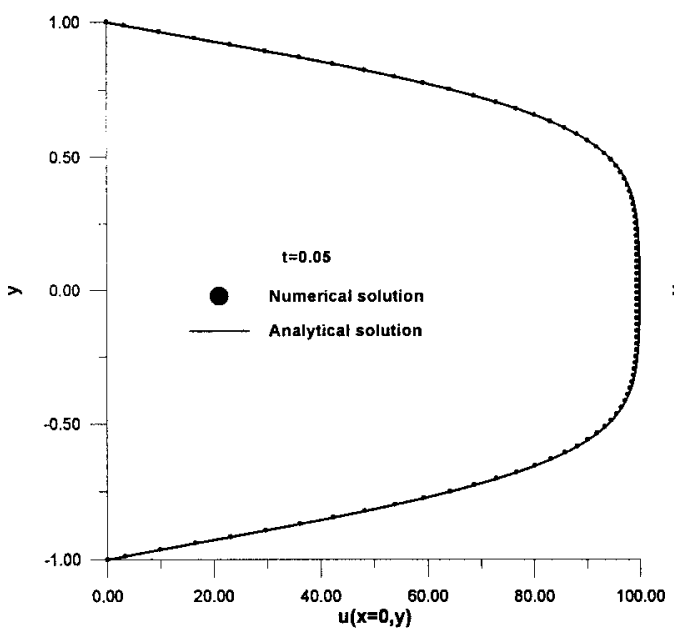

(a) $t=0.05$

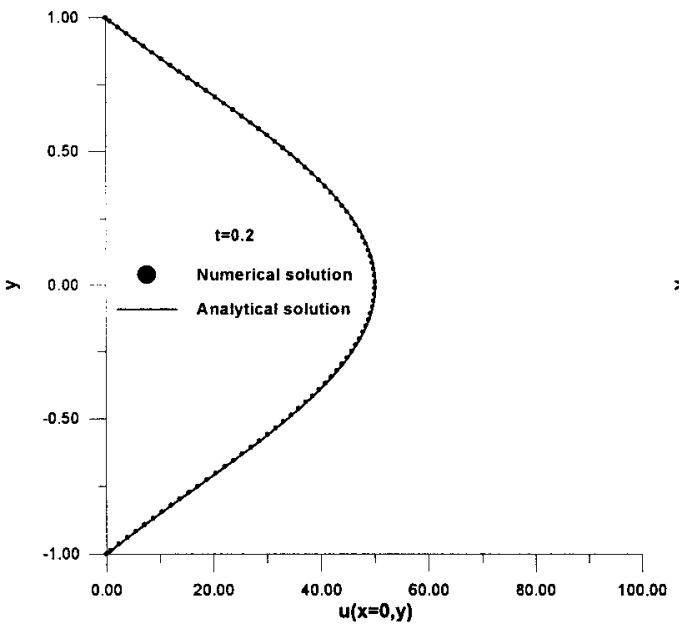

(c) $t=0.2$

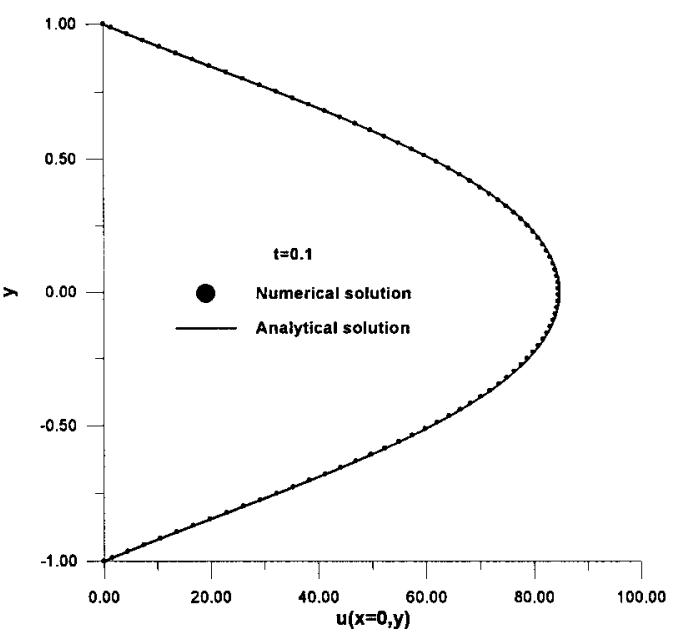

(b) $t=0.1$

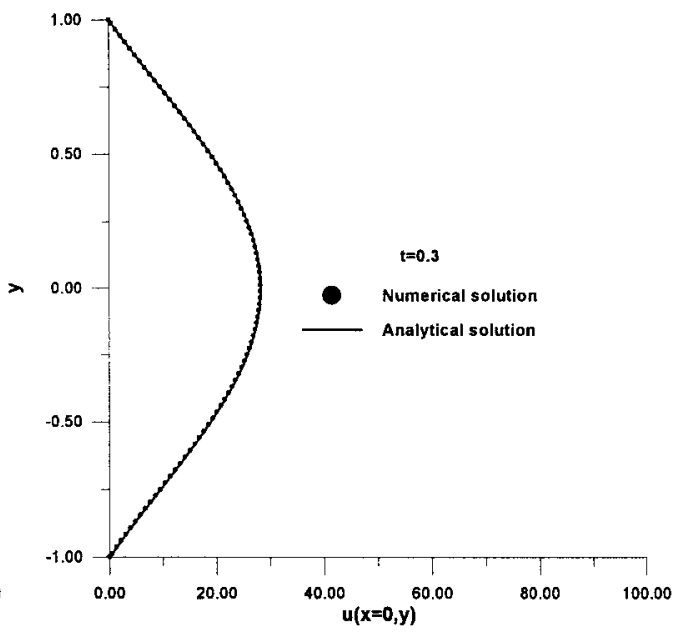

(d) $t=0.3$

FIG. 5. Comparison of u-distribution along $x=0$ by the MFS-EEM model for Example 1. (a) $t=0.05$, (b) $t=0.1$, (c) $t=0.2$, (d) $t=0.3$ (961 points, 5 eigenfunctions).

The analytical solution of the problem is given by

$$
\begin{aligned}
u(x, y, t) & =\frac{4 a b}{\pi^{2}} \sum_{m=1}^{\infty} \sum_{n=1}^{\infty} \frac{(-1)^{m+n}}{m n} \sin \left(\frac{m \pi x}{a}\right) \sin \left(\frac{n \pi y}{b}\right) e^{-\lambda_{m n}^{2} k t}+\left(x^{3}+\sin x \sin y\right) \\
\lambda_{m n}^{2} & =\left[\left(\frac{m \pi}{a}\right)^{2}+\left(\frac{n \pi}{b}\right)^{2}\right] .
\end{aligned}
$$

We set $a=1.1, b=0.9, k=1$ and use the MFS-EEM with SVD to get the first 24 eigenvalues and eigenfunctions, using 120 nodes in MFS, 961 nodes in MPS and 961 points 


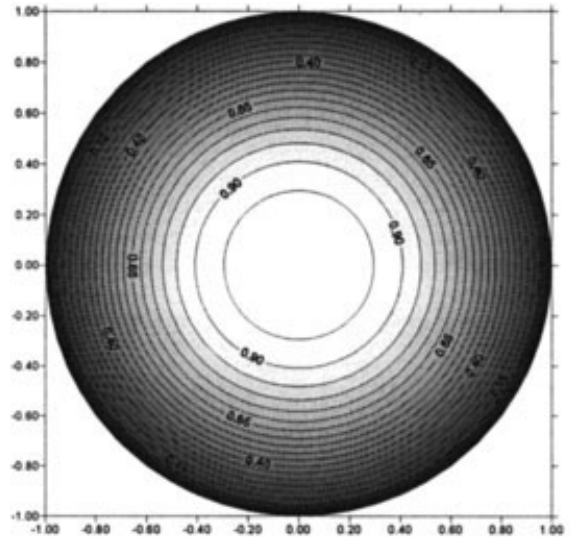

(a) $\mathrm{t}=0.05$

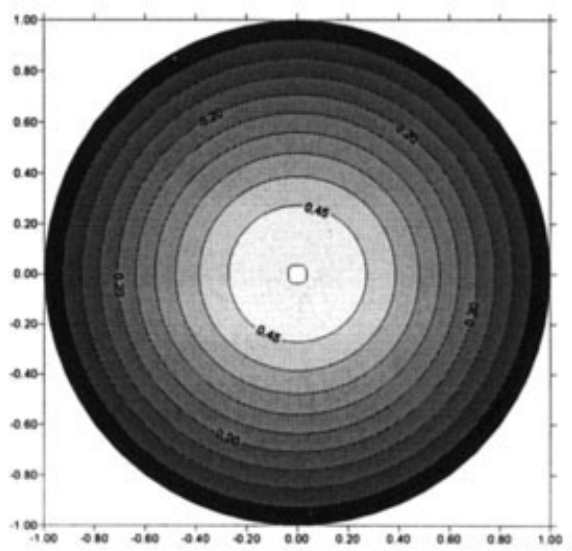

(c) $t=0.2$

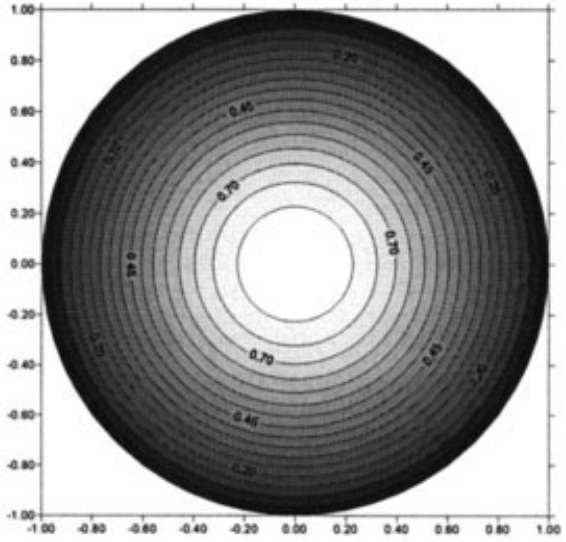

(b) $\mathrm{t}=0.1$

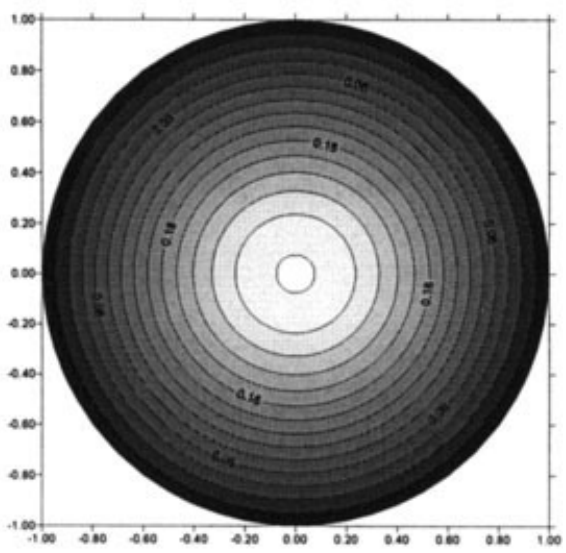

(d) $\mathrm{t}=0.3$

FIG. 6. The full-field distribution for Example 1. (a) $t=0.05$, (b) $t=0.1$, (c) $t=0.2$, (d) $t=0.3$ (961 points, 5 eigenfunctions). _ , analytical solution; - - - , numerical solution.

to interpolate eigenfunctions as displayed in Fig. 11. Again the calculated eigenvalues and the analytical solutions are very close, which demonstrates the capability of the present model to apply to different shapes of geometry. The comparisons between the present method and the analytical solution of the u-distribution along $\mathbf{x}=0.5$ are depicted in Fig. 12, when 10 eigenfunctions and 961 points to interpolate each eigenfunction are used. Almost identical results are obtained for the two solutions. And the time evolution of the full field distribution is described in Fig. 13. Except at the very beginning, the computed numerical results also show generally good agreement with the analytical solution at different time stages. The fast decay of field variation also demonstrates the physics underlying the diffusion process clearly. Figures 14 and 15 in similar fashion portray, respectively, the time evolution history of the solution at $(0.5,0.5)$, and the maximum relative error in the computational domain. On the other hand, Fig. 16 depicts the maximum relative error histogram for different numbers of points to interpolate each eigenfunction, in which more 


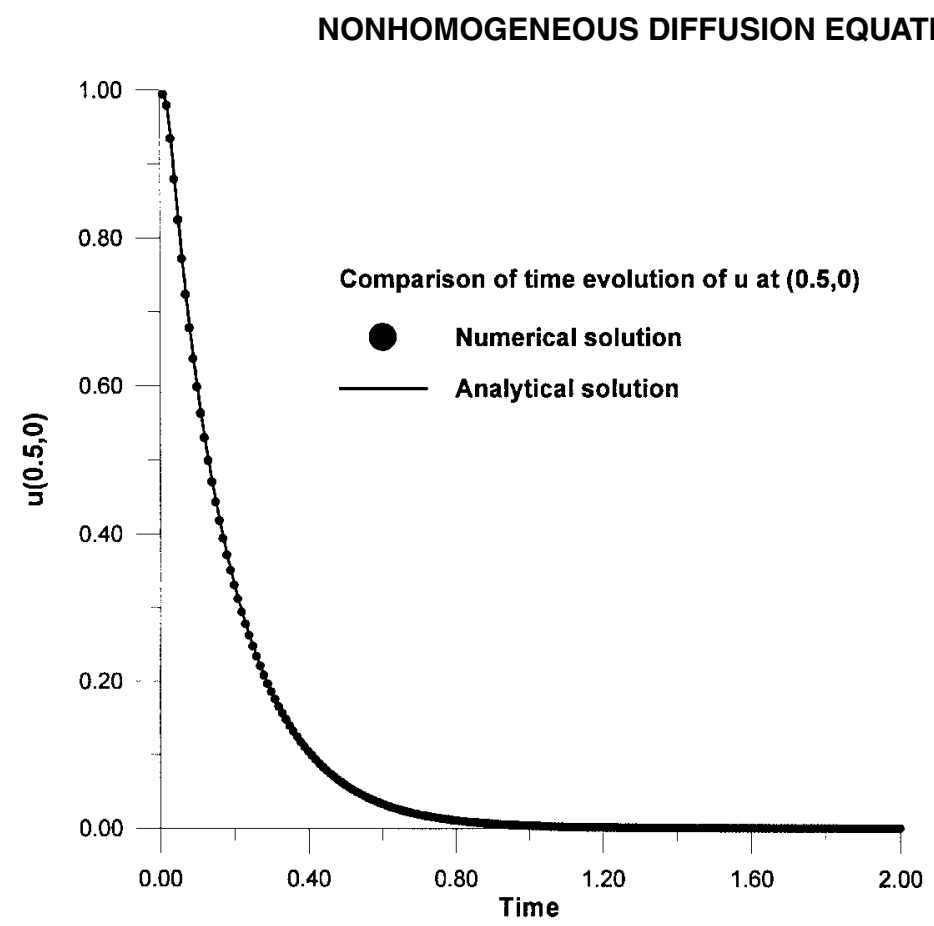

FIG. 7. Comparison of time evolution of $\mathrm{u}$ at $x=0.5, y=0$ for Example 1 (961 points, 5 eigenfunctions).

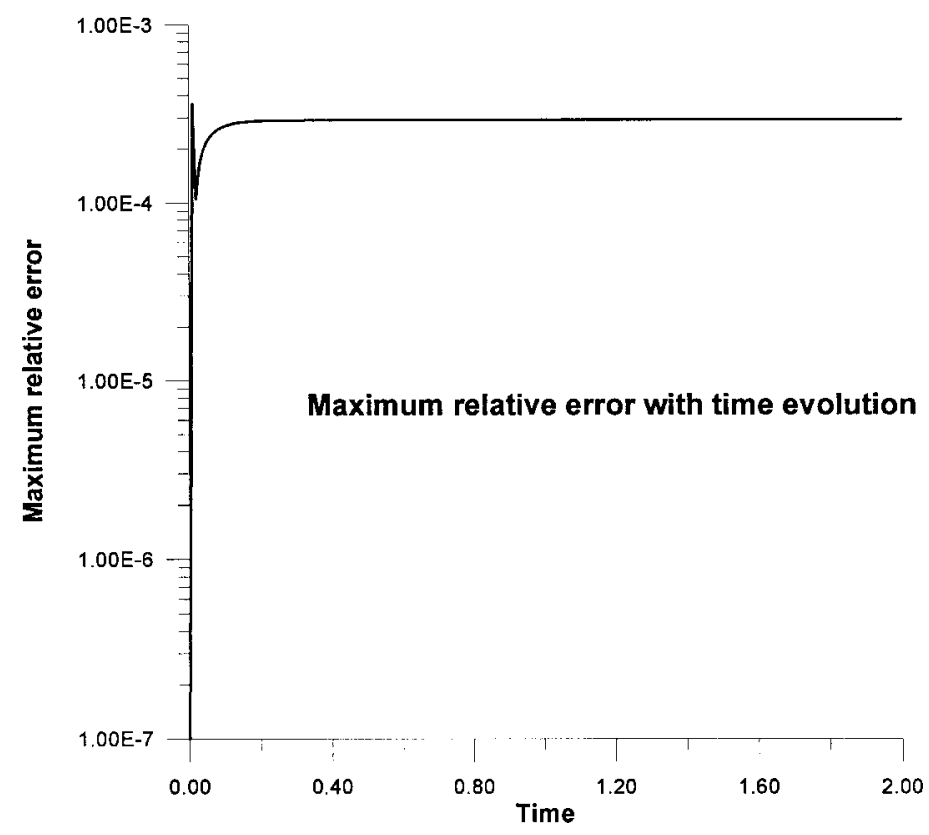

FIG. 8. Comparison of maximum relative error in the computational domain with time evolution for Example 1 (961 points, 5 eigenfunctions). 


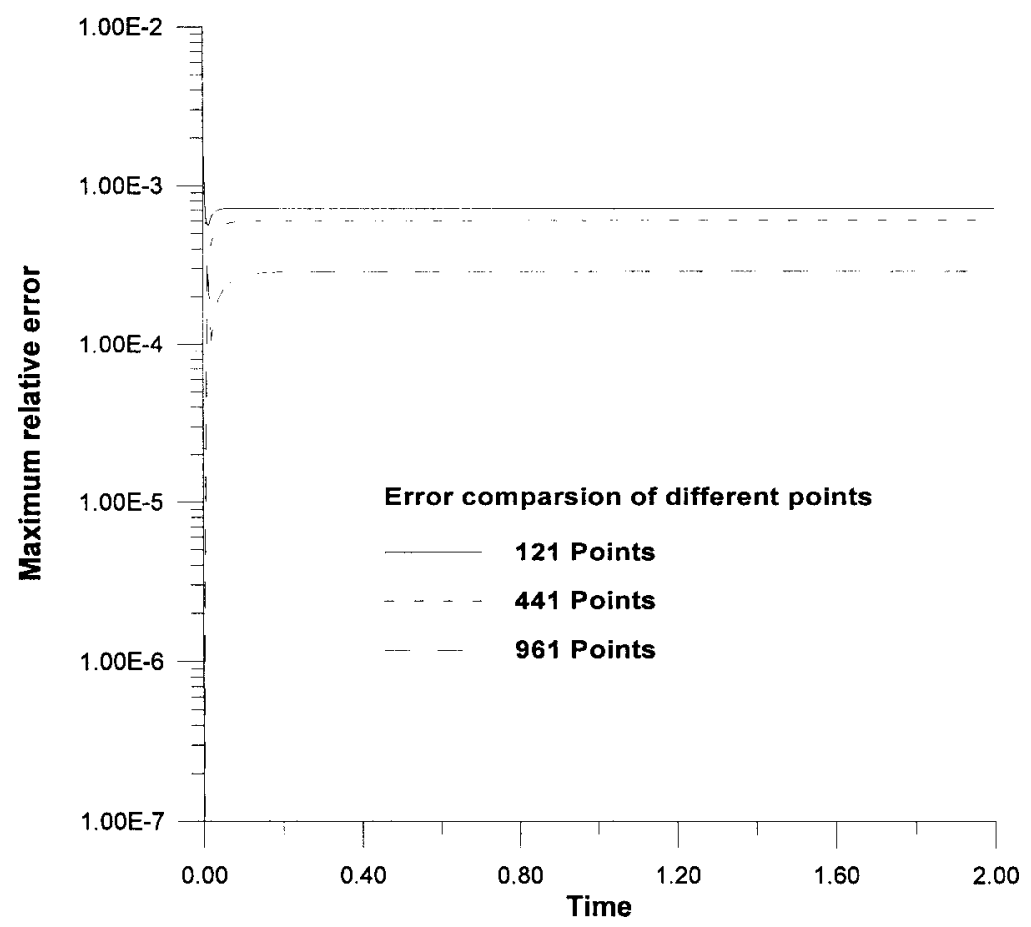

FIG. 9. Time history of maximum relative error by different points for Example 1 (5 eigenfunctions).

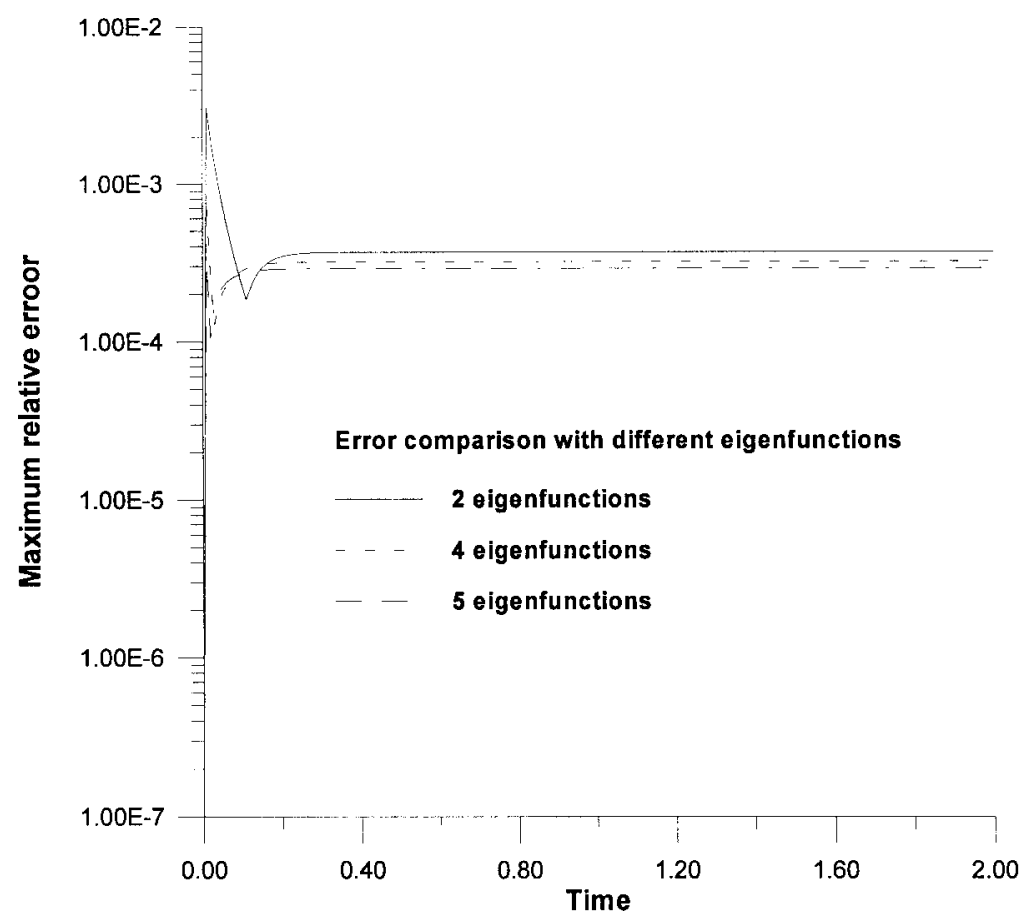

FIG. 10. Time history of maximum relative error by different eigenfunctions for Example 1 (961 points). 


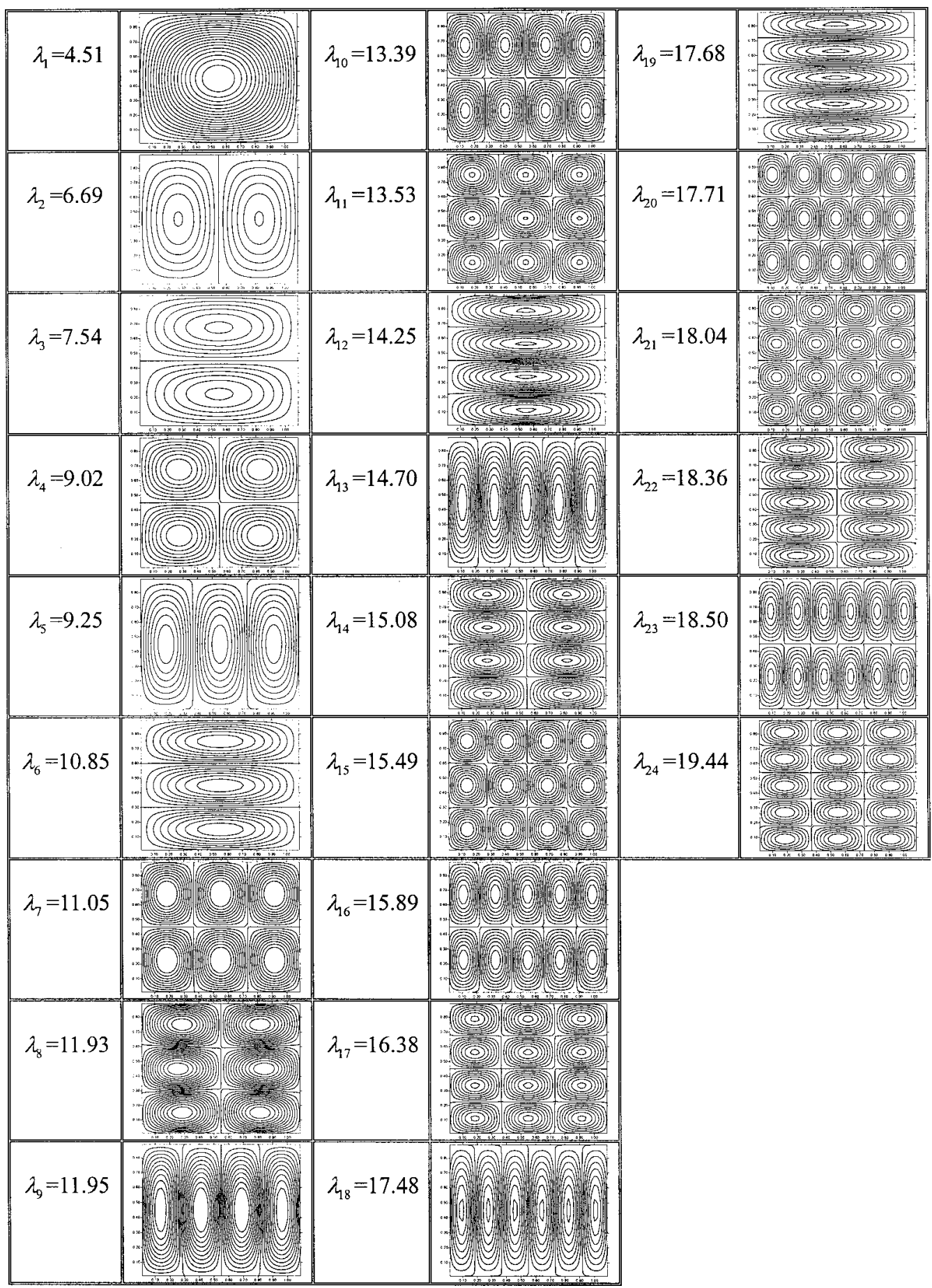

FIG. 11. The first 24 eigenvalues and eigenfunctions for rectangular problem for Example 2. 


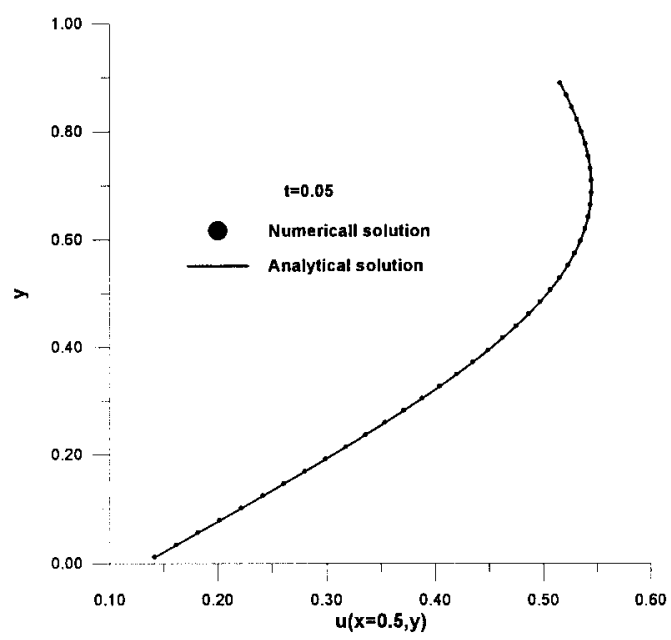

(a) $\mathrm{t}=0.05$

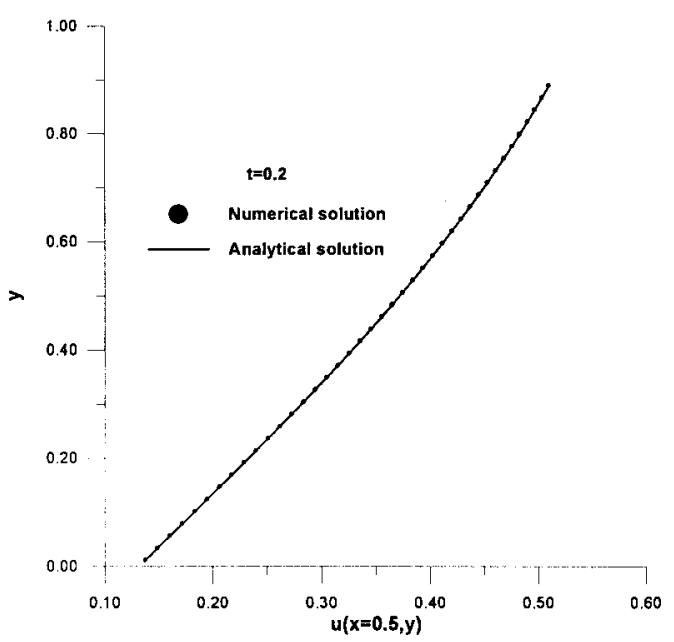

(c) $\mathrm{t}=0.2$

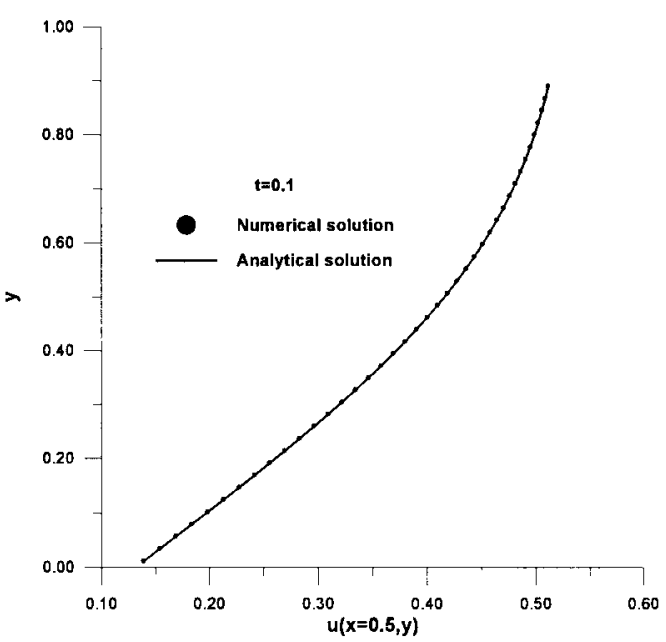

(b) $\mathrm{t}=0.1$

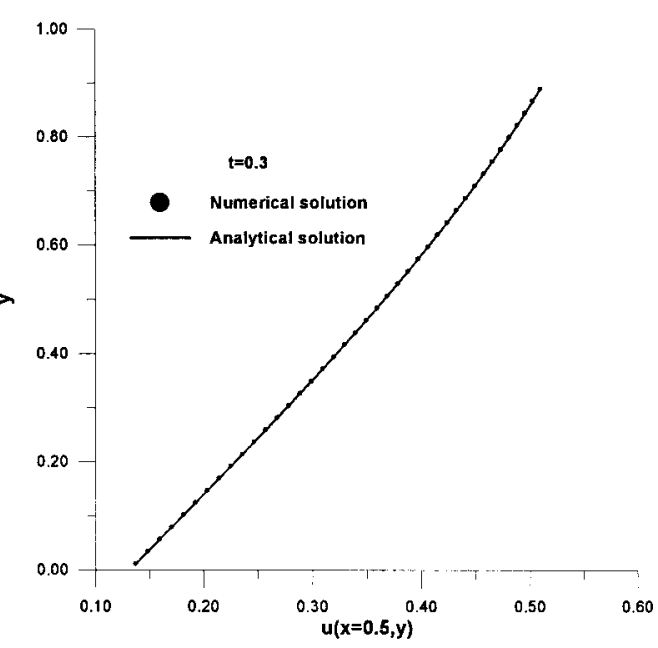

(d) $\mathrm{t}=0.3$

FIG. 12. Comparison of u-distribution along $x=0$ by the MFS-MPS-EEM model for Example 2. (a) $t=0.05$, (b) $t=0.1$, (c) $t=0.2$, (d) $t=0.3$ (961 points, 10 eigenfunctions).

points generally as expected give better results. Figure 17 shows the physical results of the diffusion problems with different numbers of eigenfunctions using 961 points to interpolate each eigenfunction, and reveals that the solution is generally acceptable.

Example 3. For the last problem, the proposed numerical method is extended to study an irregular domain, the Oval of Cassini [Fig. 3(c)], a 2D diffusion problem with nonhomogeneous boundary conditions. 


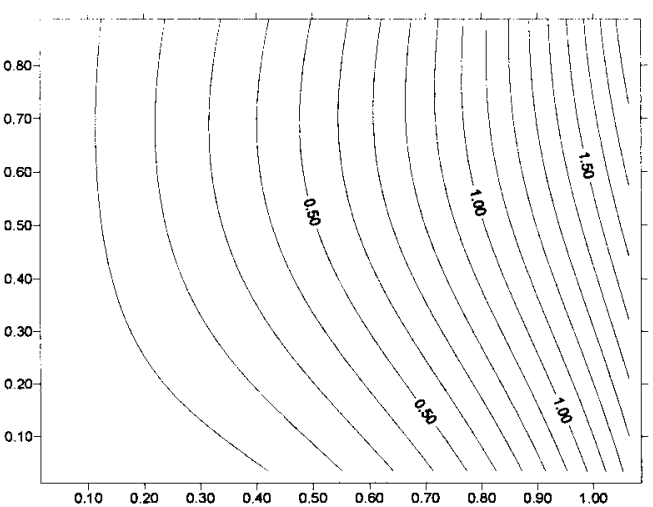

(a) $\mathrm{t}=0.05$

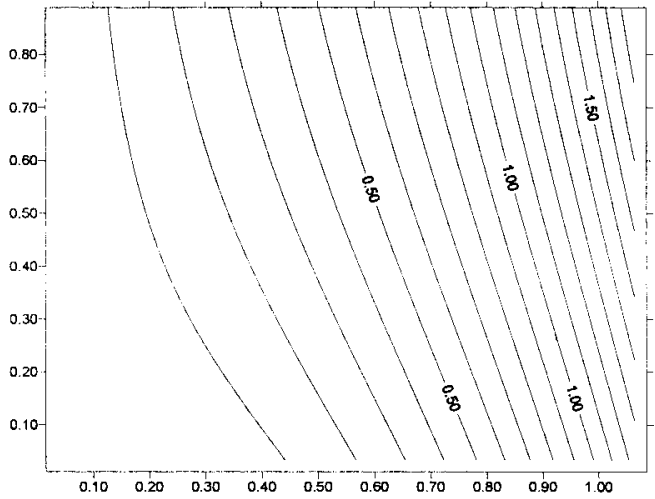

(c) $\mathrm{t}=0.2$

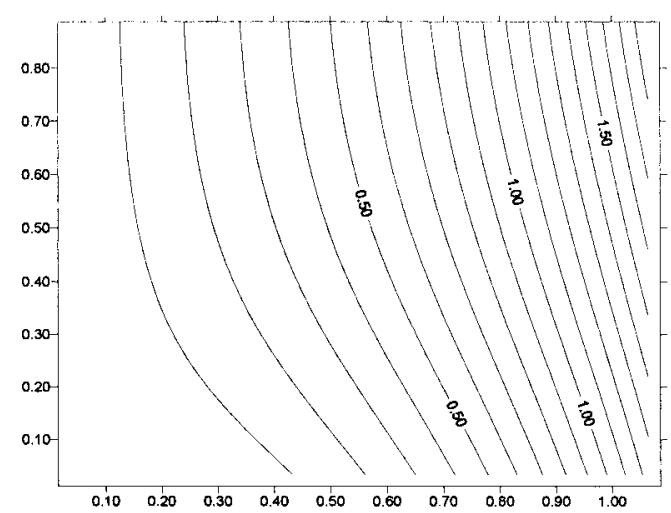

(b) $\mathrm{t}=0.1$

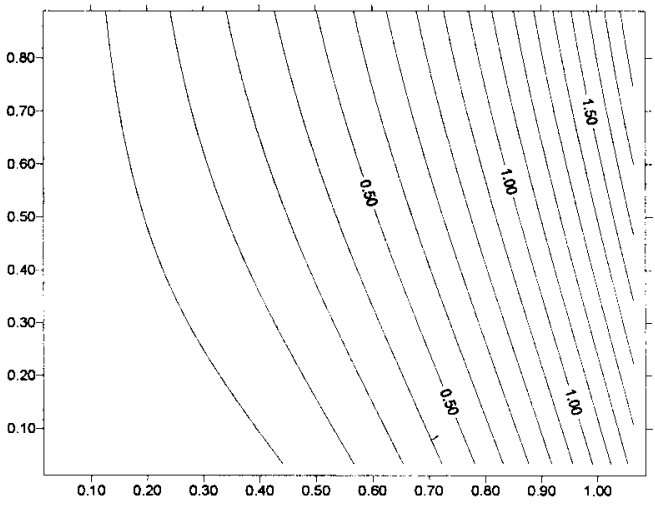

(d) $\mathrm{t}=0.3$

FIG. 13. The full-field distribution for Example 2. (a) $t=0.05$, (b) $t=0.1$, (c) $t=0.2$, (d) $t=0.3$ (961 points, 10 eigenfunctions). , Analytical solution; -- - numerical solution.

The Oval of Cassini problem can be considered as follows:

$$
\begin{array}{ll}
\Omega: & x, y \in\left[(x-1)^{2}+y^{2}\right]\left[(x+1)^{2}+y^{2}\right]=1.1 \\
\text { DE } & \left(\frac{\partial^{2} u}{\partial x^{2}}+\frac{\partial^{2} u}{\partial y^{2}}\right)=\frac{1}{k} \frac{\partial u}{\partial t} \\
\text { IC } & u(x, y, 0)=x+y \quad \text { in } \Omega \\
\text { BC } & u(x, y, t)=x+y \quad \text { on } \partial \Gamma^{1} .
\end{array}
$$

For this irregular domain, it is more difficult to get an analytical solution, so we choose FEM with unstructured grids to obtain the numerical results for comparison. The distributions of 924 unstructured FEM meshes and 80 meshless MFS nodes are described in Fig. 18. We also set $k=1$. The first 10 eigenvalues and eigenfunctions are obtained by the MFS-EEM with SVD using 160 points to interpolate eigenfunctions as displayed in Fig. 19. In similar fashion Fig. 20 portrays the time evolution history of the solutions at $(0.926,0.237)$ and $(-0.927,-0.243)$, respectively. 


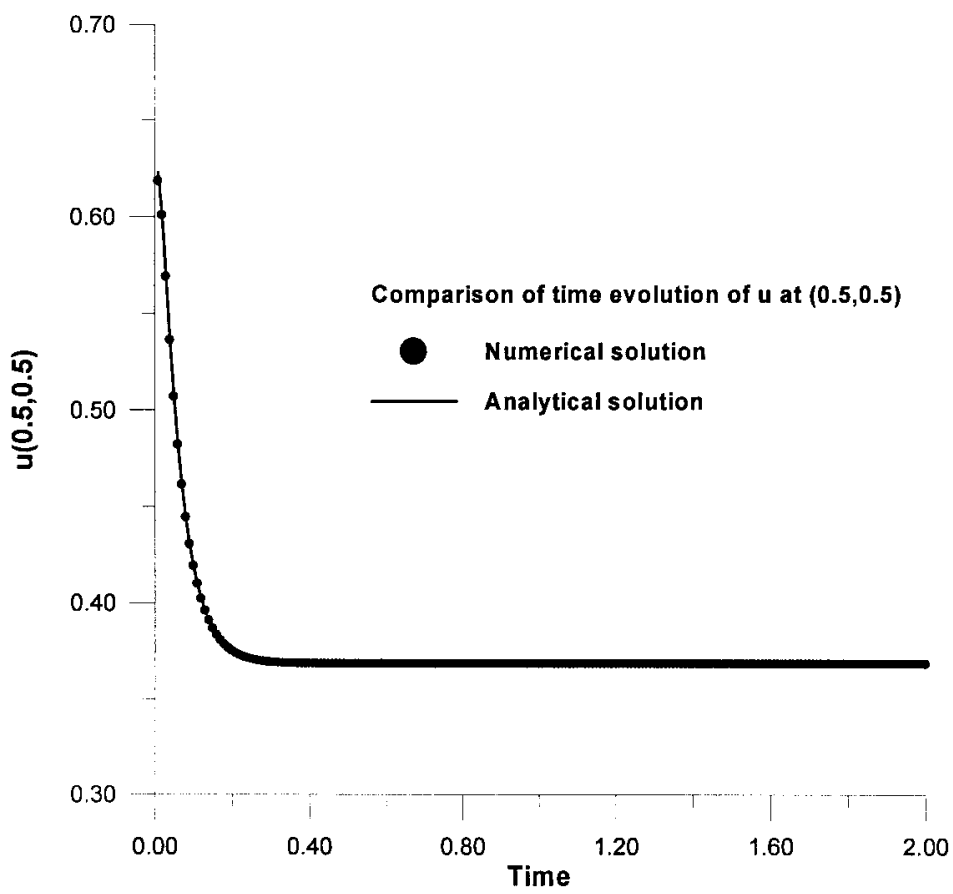

FIG. 14. Comparison of time evolution of $\mathrm{u}$ at $x=0.5, y=0.5$ for Example 2 (961 points, 10 eigenfunctions).

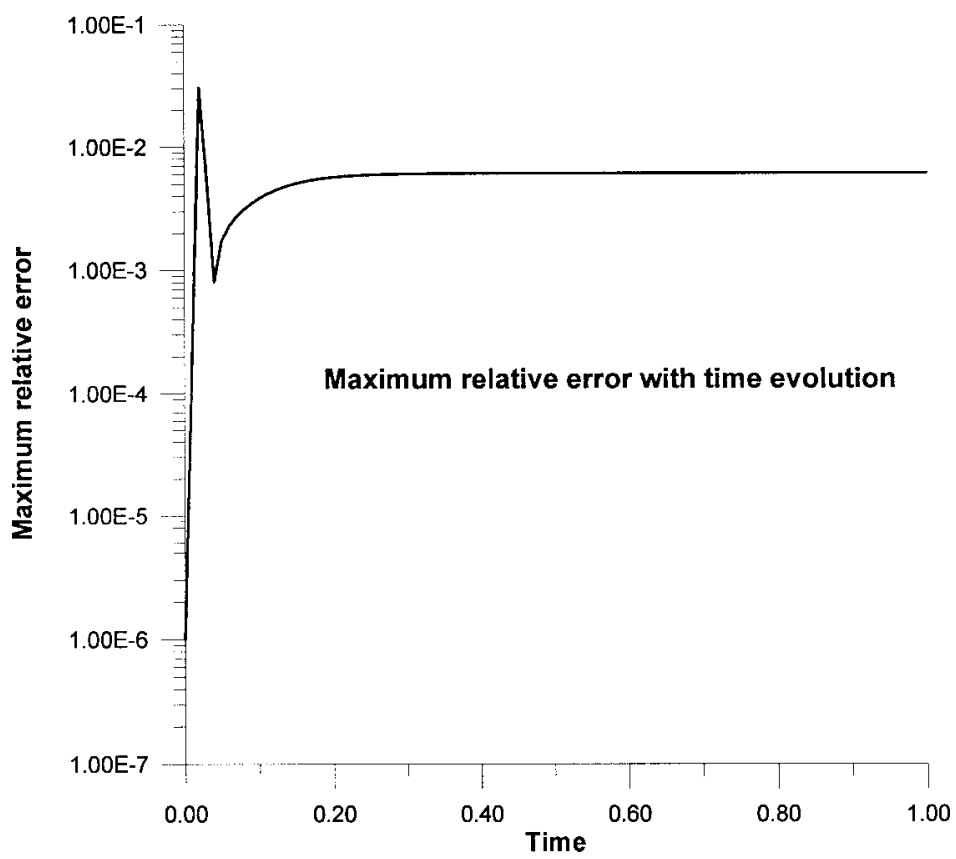

FIG. 15. Comparison of maximum relative error in the computational domain with time evolution for Example 2 (961 nodes, 10 eigenfunctions). 


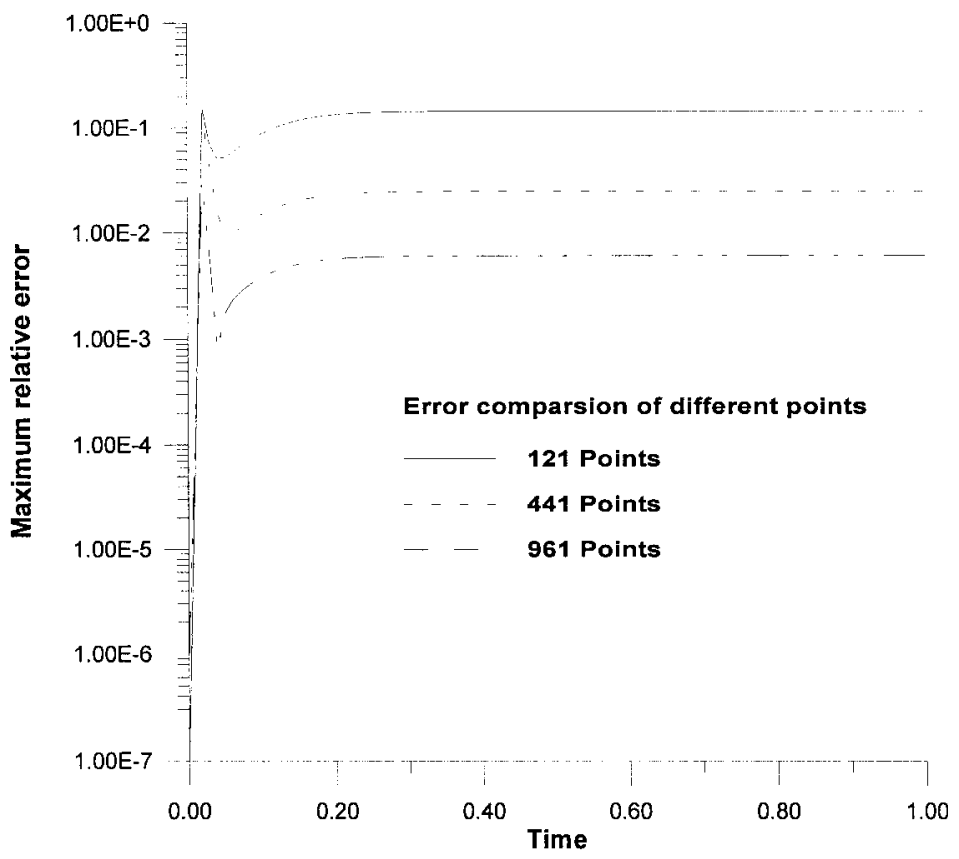

FIG. 16. Time history of maximum relative error by different points for Example 2 (10 eigenfunctions).

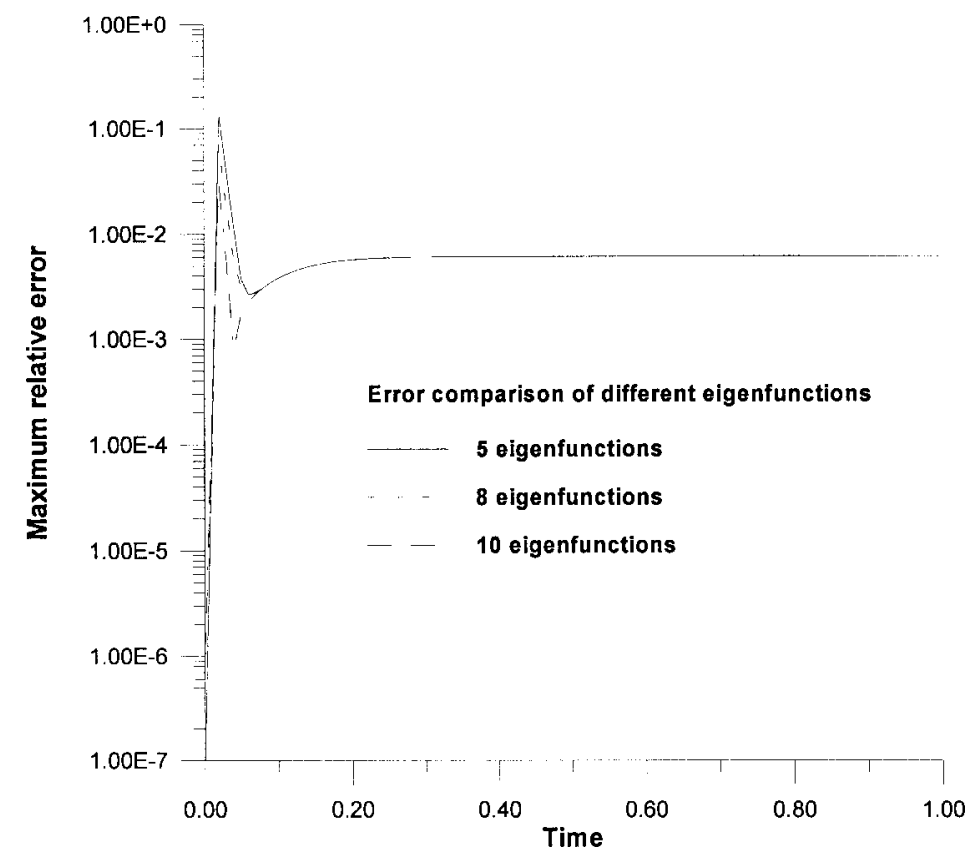

FIG. 17. Time history of maximum relative error by different eigenfunctions for Example 2 (961 points). 


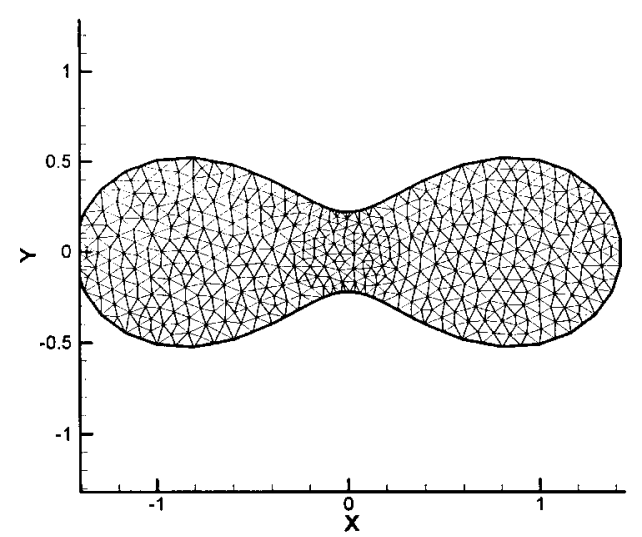

(a)

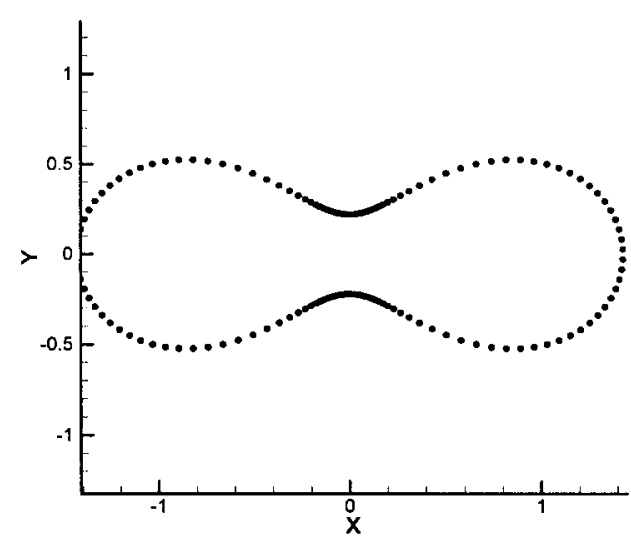

(b)

FIG. 18. (a) The unstructured meshes for the FEM (924 elements). (b) The field points for the MFS (80 nodes) for the Oval of Cassini problem for Example 3.

The results of the MFS-MPS-EEM model with the SVD method utilizing 80 nodes in MFS, 10 eigenfunctions and 160 points to interpolate eigenfunctions also match very well with the FEM solutions using 924 linear triangular elements. The present numerical method can be used appropriately even for irregular domains.

\section{CONCLUSIONS}

Transient diffusion problems with time-independent source terms and boundary conditions in multiple dimensions are solved using the MFS-MPS-EEM model together with the SVD technique. The nonhomogeneous diffusion problems with time-independent source terms and boundary conditions are separated into a Poisson equation and homogeneous diffusion equation. As far as the solution of the Poisson equation is concerned, the MFS is adopted to obtain the homogeneous solution to the Laplace equation. And the MPS is utilized to solve the particular solution due to source term of the Poisson equation. In this study the CSRBFs are adopted for the MPS. The homogeneous diffusion equation is first translated, by the EEM, into a Helmholtz equation, which is then solved by the MFS together with SVD scheme to obtain the corresponding eigenvalues and eigenfunctions.

The generally adopted Laplace transform, finite difference, and space-time collocation schemes for the time derivative term are no longer required in the proposed numerical procedure. This new algorithm has avoided time evolution methods, by properly using the superposition principle of orthogonal eigenfunctions to obtain the solutions for any time and for any given geometry. The numerical scheme developed in the present work was validated by comparison with the analytical solutions for two 2D diffusion problems under Dirichlet boundary conditions. Excellent agreements with the analytical results indicate the effectiveness of the present method to solve diffusion equations with time-independent source terms and boundary conditions without the requirement of any time transformation, discretization, or unification. The validated 


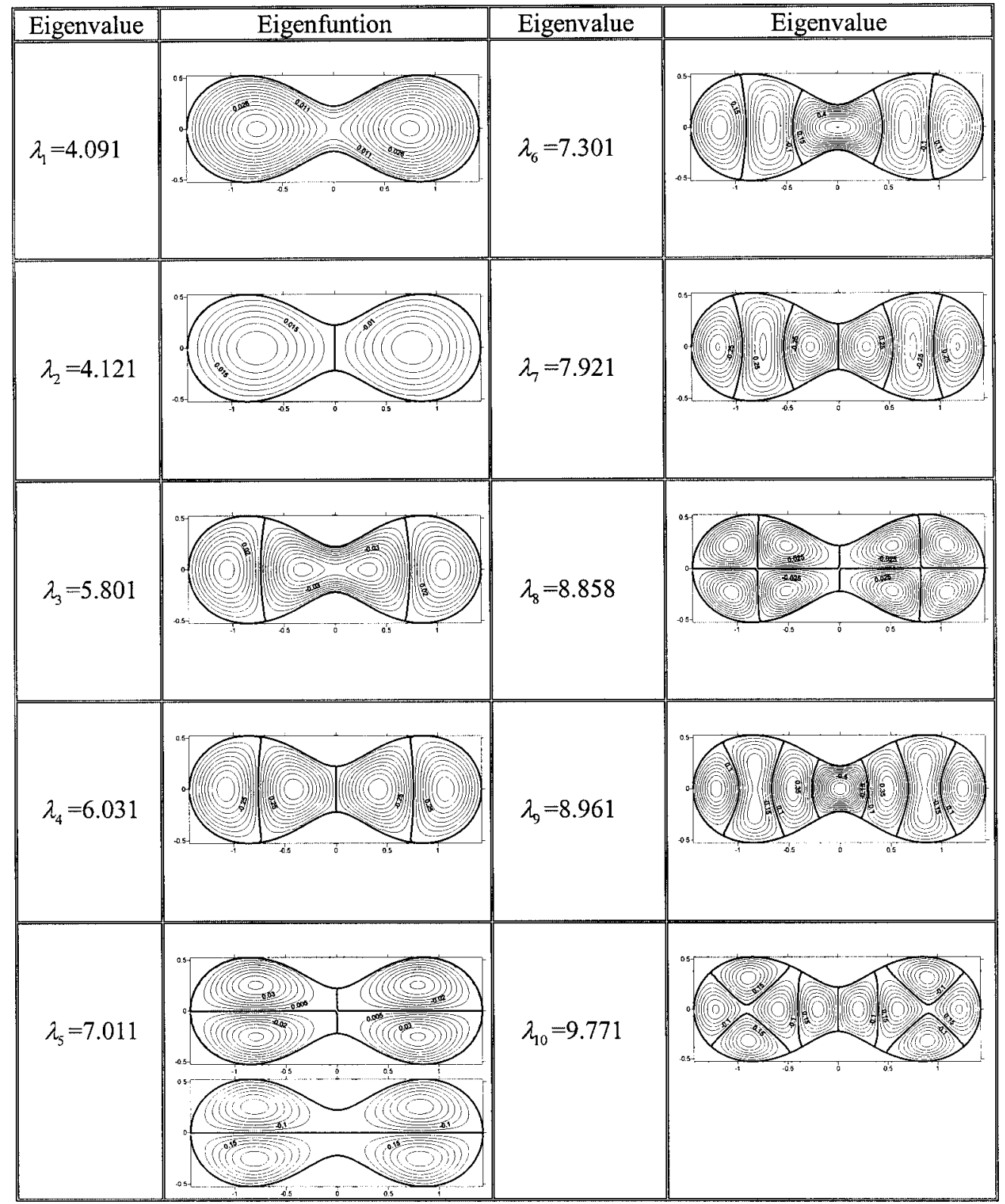

FIG. 19. The first 10 eigenvalues and eigenfunctions for the Oval of Cassini problem for Example 3.

algorithm is finally extended to an irregular domain. The results match very well with the solutions obtained by using the unstructured FEM. It is concluded that the proposed method is capable to obtain reasonable results for multidimensional nonhomogeneous diffusion equations in arbitrary domains in any time frame if time-independent source terms and boundary conditions are assumed. 
YOUNG ET AL.

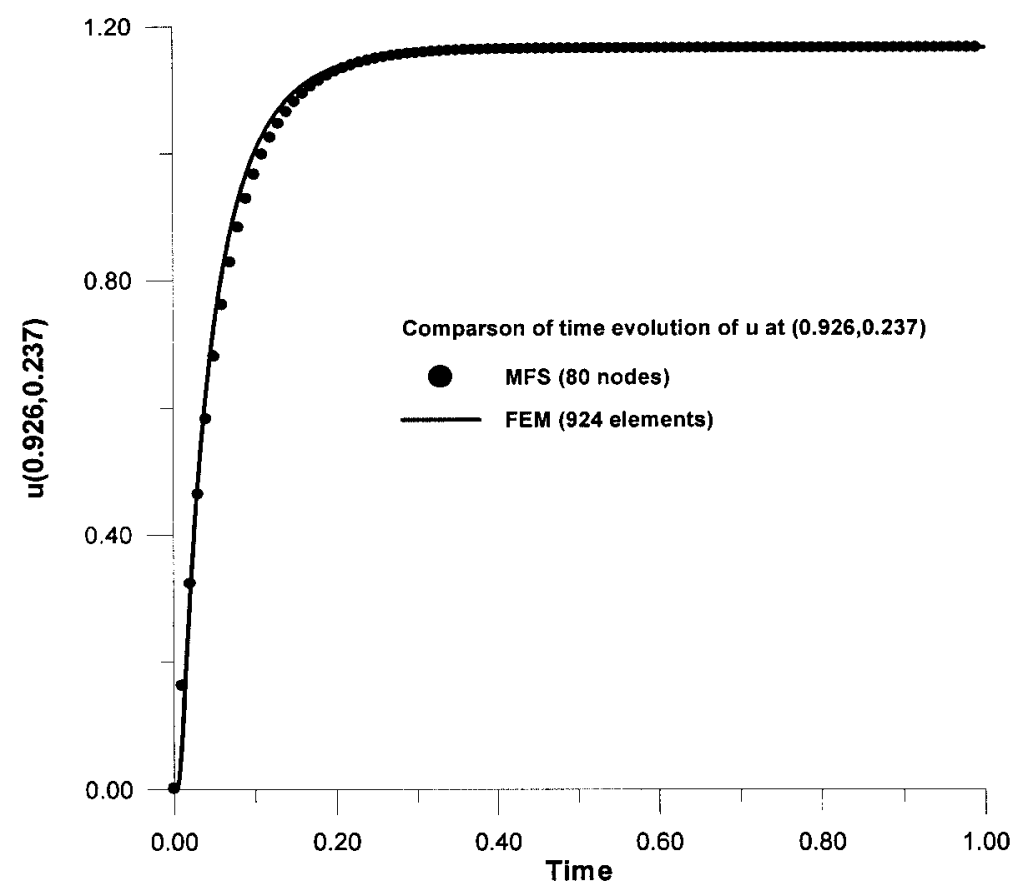

(a)

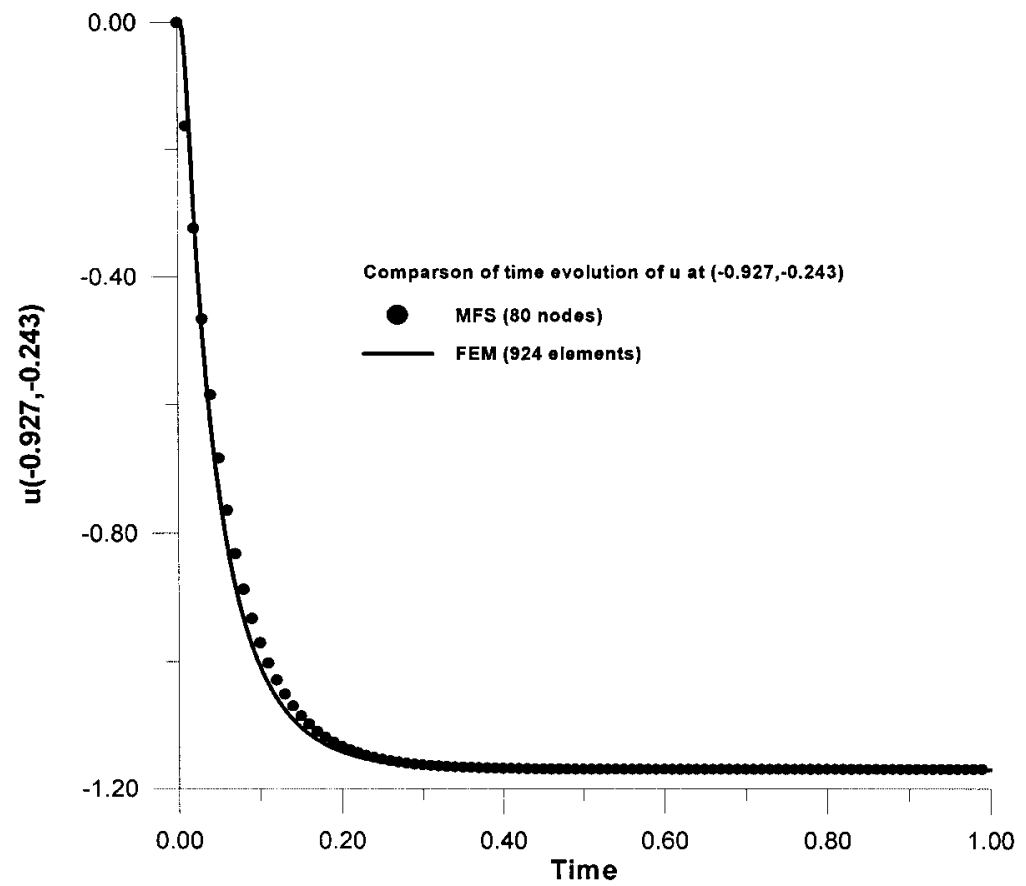

(b)

FIG. 20. Comparison of time evolution of $\mathrm{u}$ at (a) $x=0.926, y=0.237$, (b) $x=-0.927, y=-0.243$ for Example 3.

Numerical Methods for Partial Differential Equations DOI 10.1002/num 


\section{References}

1. J. M. Hobson, N. Wood, and P. J. Mason, A new finite-difference diffusion scheme, J Comput Phys 120 (1996), 16.

2. M. M. Chawla, and M. A. Al-Zanaidi, An extended trapezoidal formula for the diffusion equation, Comput Math Appl 38 (1999), 51.

3. J. T. Oden, I. Babuska, and C. E. Baumann, A discontinuous hp finite element method for diffusion problems, J Comput Phys 146 (1998), 491.

4. W. P. Jones, and K. R. Menzies, Analysis of the cell-centered finite volume method for the diffusion equation, J Comput Phys 165 (2000), 45.

5. S. P. Zhu, Solving transient diffusion problems: time-dependent fundamental solution approaches versus LTDRM approaches, Eng Anal Bound Elem 21 (1998), 87.

6. S. P. Zhu, H. W. Liu, and X. P. Lu, A combination of LTDRM and ATPS in solving diffusion problems, Eng Anal Bound Elem 21 (1998), 285.

7. V. Bulgakov, B. Sarler, and G. Kuhn, Iterative solution of systems of equations in the dual reciprocity boundary element method for the diffusion equation, Int J Numer Methods Eng 43 (1998), 713.

8. M. Zerroukat, A boundary element scheme for diffusion problems using compactly supported radial basis functions, Eng Anal Bound Elem 23 (1999), 201.

9. A. Sutradhar, G. H. Paulino, and L. J. Gray, Transient heat conduction in homogeneous and nonhomogeneous materials by the Laplace transform Galerkin boundary element method, Eng Anal Bound Elem 26 (2002), 119.

10. R. A. Bialecki, P. Jurgas, and G. Kuhn, Dual reciprocity BEM without matrix inversion for transient heat conduction, Eng Anal Bound Elem 26 (2002), 227.

11. D. Nardini and C. A. Brebbia, A new approach to free vibration analysis using boundary elements, Boundary element methods in engineering, Springer-Verlag, Berlin, Germany, 1982.

12. M. A. Golberg and C. S. Chen, The method of fundamental solutions for potential, Helmholtz and diffusion problems, Boundary Integral Methods: Numerical and Mathematical Aspects, Computational Mechanics Publications, Boston, 103-176, 1999.

13. K. Balakrishnan and P. A. Ramachandran, The method of fundamental solutions for linear diffusionreaction equations, Math Comput Modeling 31 (2000), 221.

14. C. S. Chen, M. A. Golberg, and Y. C. Hon, The method of fundamental solutions and quasi-Monte-Carlo method for diffusion equations, Int J Numer Methods Eng 43 (1998), 1421.

15. C. S. Chen, M. A. Golberg, and Y. F. Rashed, A mesh free method for linear diffusion equations, Numer Heat Transf B 33 (1998), 469.

16. D. L. Young, C. C. Tsai, K. Murugesan, C. M. Fan, and C. W. Chen, Time-dependent fundamental solutions for homogeneous diffusion problems, Eng Anal Bound Elem 28 (2004), 1463.

17. D. L. Young, C. C. Tsai, and C. M. Fan, Direct approach to solve nonhomogeneous diffusion problems using fundamental solutions and dual reciprocity methods, J Chin Inst Eng 27 (2004), 597.

18. Z. Yao, and G. F. Margrave, Wavefield extrapolation in laterally inhomogeneous media by generalized eigenfunction transform method, CREWES Research Report 11, 1999.

19. J. T. Chen, H.-K. Hong, C. S. Yeh, and S. W. Chyuan, Integral representations and regularizations for a divergent series solution of a beam subjected to support motions, Earthquake Eng Struct Dyn 25 (1996), 909.

20. C. S. Chen, and R. A. Schaback, Recent developments of the dual reciprocity method using compactly supported radial basis functions, Transformation of the domain effects to the boundary, Y. F. Rashed, editor, WIT Press, 2002. 
21. W. Madych, Miscellaneous error bounds for multiquadric and related interpolants, Comput Math Appl 24 (1992), 121.

22. C. S. Chen, C. A. Brebbia, and H. Power, Dual reciprocity method using compactly supported radial basis functions, Commun Numer Methods Eng 15 (1999), 137.

23. A. H.-D. Cheng, D. L. Young, and C. C. Tsai, Solution of Poisson's equation by iterative DRBEM using compactly-supported, positive-definite radial basis function, Eng Anal Bound Elem 24 (2000), 549.

24. D. L. Young, C. C. Tsai, and A. H.-D. Cheng, Solution of Stokes flow using an iterative DRBEM based on compactly-supported, positive-definite radial basis function, Comput Math Appl 43 (2002), 607.

25. P. A. Ramachandran, Method of fundamental solutions: singular value decomposition analysis, Commun Numer Methods Eng 18 (2002), 789. 